\title{
Téoros
}

Revue de recherche en tourisme

\section{Le tourisme dans la vie des nouveaux musées de Montréal : un premier tour d'horizon}

\section{Marc Laplante et Marie-Janou Lusignan}

Volume 11, numéro 2, juillet 1992

Quand les musées s’ouvrent au tourisme...

URI : https://id.erudit.org/iderudit/1078088ar

DOI : https://doi.org/10.7202/1078088ar

Aller au sommaire du numéro

Éditeur(s)

Université du Québec à Montréal

ISSN

0712-8657 (imprimé)

1923-2705 (numérique)

Découvrir la revue

Citer cet article

Laplante, M. \& Lusignan, M.-J. (1992). Le tourisme dans la vie des nouveaux musées de Montréal : un premier tour d'horizon. Téoros, 11(2), 19-25.

https://doi.org/10.7202/1078088ar d'utilisation que vous pouvez consulter en ligne. 


\section{Le tourisme dans la vie des nouveaux musées de Montréal : un premier tour d'horizon

\author{
Marc Laplante et Marie-Janou Lusignan*
}

Le portrait muséologique de Montréal a changé drastiquement au cours des premiers mois de 1992 après avoir été quasiment immobile pendant une vingtaine d'années. L'an passé, le Centre d'histoire de Montréal réouvrait ses portes après une rénovation majeure et, il y a trois ans à peine, le Centre canadien d'architecture apportait une grande nouveauté dans l'équipement culturel montréalais. Le $350^{\circ}$ amiversaire de Montréal a certainement servi d'occasion pour inaugurer plusieurs nouveaux musées cette année: le Biodôme, le Musée d'art contemporain, le Musée d'archéologie et d'histoire de la Pointeà-Callière et pour réouvrir des musées ou des centres d'exposition entièrement rénovés et agrandis: le Musée des Beaux-Arts, le Musée MoCord et la Palais de la Civilisation.

Téoros, en consacrant un numéro aux relátions entre le tourisme et les musées, se devait de traiter spécialement de ces nouvelles institutions muséales et patrimoniales de Montréal. A priori, nous avons imaginé que ces grands musées, nouveaux ou largement rénovés, avaient eu toutes les chances de profiter des nouvelles connaissances en tourisme pour adopter leurs aménagements physiques et leurs programmations en fonction de ces clientèles. Les 6 institutions mentionnés cihaut ont certainement pensé au tourisme et aux touristes en concevant leurs bâtiments, en développant leurs installations et leurs services, en préparant leurs plans de marketing, etc. Nous avons donc préparé un court plan d'entrevue pour aborder ces questions avec les responsables de ces muscés et centres d'interprétation. Ces entrevues se sont faites entre le 25 juin et le 6 juillet demier, c'est-ă-dire presqu'a leur ouverture, pour certains musces, et déjà en haute saison touristique pour tous.

Les pages qui suivent présentent un portrait rapide de la place du tourisme dans la vie de 6 musées montréalais récemment inaugurés ou

Monsieur Marc Laplante est professeur au departement d'etudes urbaines et touristiques de IUDAM: il a réaliséles entrevues au Biodône, au Musée des BeauxArts et a la Pointe-a-Calière. Madame Marie Janou Lusignan est étudiante à la maitrise en musbologie [UDAM et Université de Montréal] et diplamee en gestion et intervention touristiques de l'UOAM; elle a réaliske les autres entrevues. réouverts au public. Les deux auteurs ont choisi de rendre compte de leurs échanges avec les responsables interrogés sans entreprendre de bilan ni de comparaison. Les lecteurs ne manqueront pas de relever toutefois quelques contrastes qui peuvent s'expliquer facilement à première vue: certaines institutions, comme le Muś́e des Beaux-Arts et le Palais de la Civilisation, ont déjà une longue expérienoe de projets conjoints avec les intervenants touristiques; a l'opposé, une institution aussi nouvelle que le Muscé de Pointe-àCallière doit tout faire pour initier ses rapports avec l'industrie touristique.

Pour certains, ces portraits de situation sembleront trop rapides. On s'étonnera peut-êture de ne pas trouver de résistance ou d'attitudes réservées ou sceptiques à propos du tourisme dans ces comptes-rendus: dans tous les cas, on șest dit intéressé à accueillir des touristes et à metre en ceuvre les moyens nécessires pour les satisfaire le mieux possible; presque dans tous les cas également, les touristes ne sont pas perçus comme une clientele nettement differente des autres visiteurs. Enfin, même si certains responsables ont souligné que les touristes n'avaient pas, en arrivant au musée, un bagage de connaissances semblable à celui d'un Montréalais ou d'un Québécois, ce fait ne semble pas poser de problemes particuliers et peut être assumé par l'action des guidesanimateurs ou par quelques supports particuliers d'interprétation.

$\mathrm{Si}$, comme on le dit parfois, les relations entre le tourisme et la culture sont difficiles, on ne peut pas endosser ce point de vue a partir des témoignages qu'on lira ci-dessous.

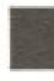

\section{Biodôme de Montréal}

Madame Johanne Landry
Responsable des programmes
publics et educatifs
Madane Francine Gerwais
Communication-marketing

Le Biodôme n'a pas eu à prévoir des aménagements très speciaux pour les touristes. Sa première clientèle est celle du Jardin Botani- que et, dès le début du projet, il fut convenu qu'un billet d'entrée serait disponible pour accéder à la fois au Biodôme et au Jardin Botanique (coût: 12 \$, valide pour deux jours et assurant une entrée sans attente au Biodôme). Le fait également que le Biodôme soit une installation du Parc Olympique est un atout certain du point de vue touristique puisque ce Parc dispose maintenant d'aménagements spécialisés pour accueillir les touristes.

Madame Landry a abordé ensuite la question du bilinguisme comme élément de politique touristique: les panneaux d'interprétation sur les sentiers des quatre écosystèmes du Biodôme sont en français et en anglais, les animateurs spécialisés en sciences naturelles (18 personnes) sont aussi capables de communiquer dans ces deux langues, des documents écrits existent en version française et anglaise et la signalisation sera au moins bilingue sinon trilingue - quand tout sera terminé l'an prochain.

Pour comprendre et apprécier à sa juste valeur la richesse des éléments mis en exposition au Biodồne, le visiteur doit y consacrer du temps. Le sujet exposé est notre planète! Onne saisira ce tout complexe, minéral, végétal et animal, qu'en prenant le temps de voir les nombreuses interactions entre ces éléments dans chacun des quatre écosystèmes composant le Biodôme: la forêt tropicale, la forêt laurentienne, le Saint-Laurent marin et le monde polaire.

Comment sensibiliser et ensuite soutenir l'intérêt des visiteurs dans un tel musce qui expose du vivant sans le détacher de son milieu naturel de vie (a l'encontre de la majorité des Jardins botanique et zoologique)?

Le Biodồne s'est préoccupé de cette tîche éducative dès sa création. Ainsi, au Carrefour de l'environnement, situé au centre des quatre écosystèmes, le public est préparé à sa visite par des images, par des animateurs et par des périodes de questions pour que chacun sache le mieux possible ce qu'il ira visiter. Il existe aussi des kiosques d'informations près de la billetterie; pour compléter une visite, on a créé Naturalia, un espace interactif où les gens peuvent toucher, manipuler, expérimenter des 


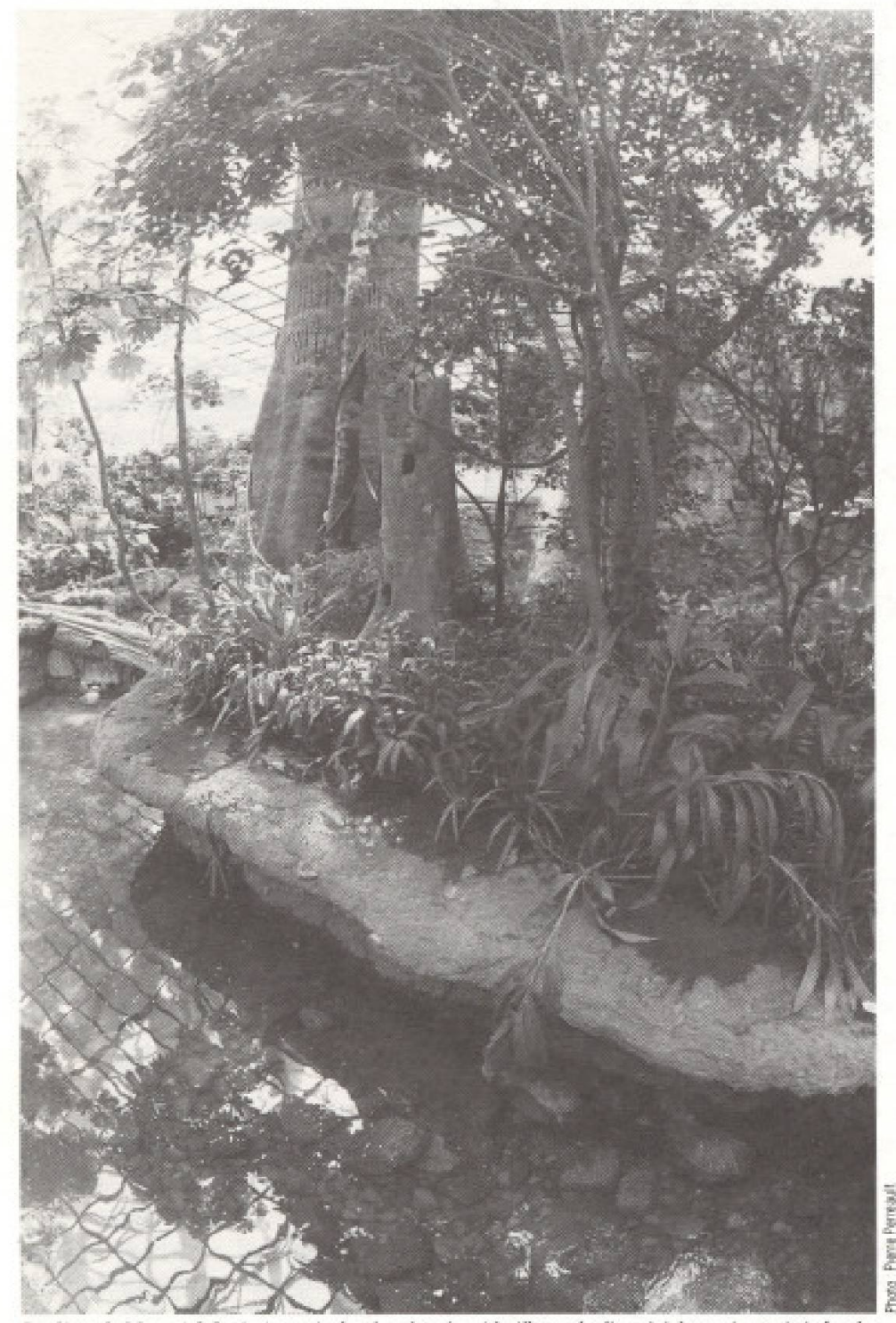

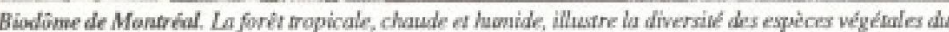
bassin anusonien.

objets qui mettent en relief diverses fonctions adaptatives des animaux et des végétaux.

Ainsi prépartés et encadrés, les visiteurs s'engagent sur les sentiers des 6cosystèmes. Ils peuvent commencer par celui qui les attire le plus et passer d'un écosystème à un autre en suivant la signalisation ou en revenant au Carrefour après la visite d'un écosystème donné pour s'engager dans les sentiers d"un autre écosystème de leur choix. L'idée centrale de cette organisation des visites est de permettre à chacun d'y aller selon son rythme; on suggère constamment aux visiteurs de prendre les jumelles, des'attarder à l'observation, d'interroger les animateurs disséminés partout sur les sites.

Le Biodôme n'offre pas de visites guidées au sens courant de ce teme (un guide accompa- gnant un groupe sur le site et faisant l'interprétation). Malgré l'immensité de l'ancien vélodrome, les éléments de chacun des écosystèmes sont si abondants qu'il a fallu réduire les espaces de visites à des sentiers principalement (et à quelques belvédères pour voir des panoramas). Dans ces sentiers, on voit mal comment des groupes de visiteurs pourraient se déplacer, en tant que groupes.

Est-ce à dire, en fin de compte, que le touriste, en tant que touriste, n'a fait l'objet d'aucune attention spéciale pour qu'il puisse profiter de cette altraction exceptionnelle à Montrual? Certes non, mais il $y$ avait un défí à relever car un site comme le Biodôme c'est un peu comme le Louvre: il faut y revenir souvent pour tout voir et suriout, tout apprécier (par exemple, les quatre saisons de la forêt laurentienne). Or, par définition (ou presque), le touniste manque de temps! Le Biodôme en une heure?

Madame Landry n"a pas été décontenancée parr cette question. Elle et son équipe ont préparé des outils et des moyens pour que des groupes puissent bénéficier de l'essentiel du Biodôme en 60 minutes environ. Une stagiaire en muséologie, madame Annie Lafrance, a préparé un scénario de visite commentée du Biodône de Montréal (décembre 1991). Ce texte est destine aux guides touristiques pour les aider à péparer leurs groupes avant de commencer la visite et à les soutenir à certains endroits précis (sur les belvédères) le long des sentiers. Même s'il est certain que cee document sera revu après rodage, son existence, avant même l'ouverture officielle du Biodôme, témoigne de l'effort d'analyse et de réflexion des responsables des publics: pas des concessions exagérées pour le public touristique quant aux objectifs originaux du projet: la vulgarisation scientifique, mais respect des conditions particulières de visite de chaque catćgorie de visiteurs. Et madame Landry, en terminant, a bien rappelé que son groupe favori de visiteurs est une famille avec enfants, groupe pour lequel on peut à la fois s'occuper de rigueur scientifique et de pédagogie. Le Biodôme prolonge donc parfaitement le Jardin botanique en le complétant car, à part l'Insectarium, ce Jardin botanique ne peut pas facilement intéresser les 12 ans et moins.

Madame Landry a traité plus rapidement des questions relatives à la commercialisation du produit touristique du Biodôme. II existe déja des forfaits pour le Jardin botanique qui incluent maintenant le Biodôme; des forfaits avec la Planétarium sont à venir, il y a des demandes pour des forfaits incluant le Pare Olympique (visite du stade, ascension du mât, etc.) et le Biodôme. Selon notre informatrice, il y a wolonté de part et d'autre de créter de tels produits et il reste à trouver une manière de le faire. Enfiñ, dès l'an prochain, le Biodôme sera plus présent aux grands moments de l'industrie touristique (salons, market places, etc). Le déjà célêbre Biodôme a l'avenir pour lui.

\section{Pointe-à-Callière \\ Musée d'archéologie et d'histoire de Montréal}

\section{Madame Francine Lelièvre Directrice générale}

Le tout nouveau Musée d'archéologie et d'histoire de Montráal à la Pointe-à-Callière sera sûrement le point de départ obligé des visites touristiques qui chercheront à bien situer Montréal dans l'histoire de l'Amérique et du monde.

Le Musée de la Pointe-â-Callière est consacré d'abord à la découverte de Montráal comme ville de commerce et d'échanges. Le site choisi parr Maisonneuve était déjà un lieu d"échanges pour les sociétés amérindiennes avant l'arrivée des Européens. Une projection dans une salle multimédia unique en son genre au monde, affïrne la directrice générale, parce qu'elle se trouve installée sur les fouilles archếologiques mêmes, rappelle vite et bien comment ce site de Montrual ctait un endroit stratégique pour toutes activités de transports. 
Les amateurs d'histoire et d'archéologie apprécieront ce court spectacle audio-visuel qui fait une bonne synthèse de l'histoire de Montrél et introduit clairement au reste du musée.

Car l'aventure commence. Du nouvel édifice de l'Epéron, sur la Pointe-ât-Callière, le visiteur descend dans le crypte (ou les catacombes) qui s'étend sous la place Royale jusqu'à l'édifice de l'Ancienne Douane (rue StPaul) et qui se prolonge dans l'axe du lit de l'ancienne rivière Saint-Pierre. Ces vestiges permettent d'interpréter les couches sucoessives de l'histoire de Montréal qui s'est vécue là, surce site de la place Royale et de ses environs. Le travail des archéologues est manifeste, les vestiges sont animés de diverses façons (nombreuses maquettes de la place Royale à diverses cpoques sous un plancher de verre, interrogation de personnages fautômes qui répondent à vos questions sur tel ou tel édifice ou événement, guides spécialisés qui népondent aussi à vos questions et qui parfois, pour un petit groupe, feront une capsule d'interpritation historique fort vivante, etc.

Cette descente dans les couches d'histoire de Montréal est une expérience assez particulière. A premiere vue, on peut croire que seul un public assez familier avec l'archéologie $s^{3}$ attardera à parcourir ces dédales de l'histoire. Mais les concepteurs du Musét n'ont pas hésité à metre en oeuvre divers moyens pour rendre cette visite souterraine intéressante. Par exemple, pour mieux rappeler la rivière canalisée au XIXe siècle, là où coulait jadis la rivière St-Pierre, un écran lumineux repressente ce que devait être la rive du fleuve St-Laurent à l'endroit où la canalisation voùtée rejetait les eaux usces de la ville dans le fleuve.

Enfin, troisième élément de ce complexe muséal, la visite souterraine se termine sous l'édifice de l'ancienne douane, belle construction de 1836 qui loge maintenant, au rez-dechaussce, une très intéressante boutique et, à l'étage, une exposition permanente originale intitulée $D u$ wampum (monnaie amérindienne) à la carte de crédit. Montréal, carrefour d'échanges ei de conmerce. Cette exposition reprend intelligemment le theme des couches d'histoire exploité en sous-sol pour parler de la Ville de Montréal d'aujourd'huí comme résultante d'une histoire.

Voila donc le fait unique de ce musée: il permet au visiteur de suivre a la trace, sur les lieux mêmes de l'histoire, les 350 ans de Montréal qui ont commencé sur un site déjà

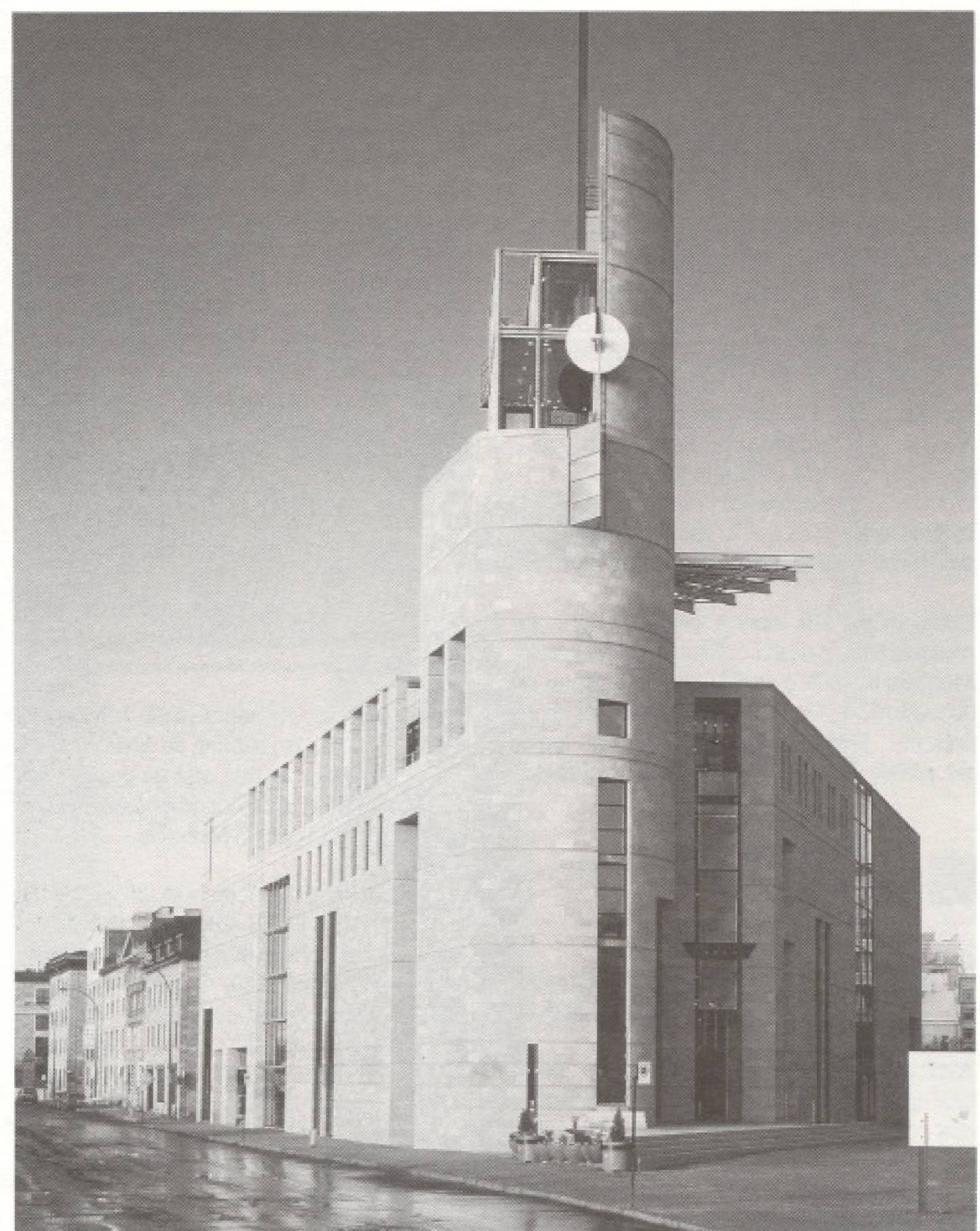

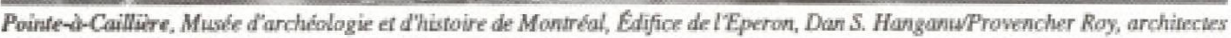

important pour les échanges et le commerce entre les premieres nations.

Comment, dans cette installation muséale complexe, le touriste va-t-il se retrouver et faire une expérience inoubliable? Madame Lelièvre fut, dès les premières heures du projet, une conceptrice qui avait nettement conscience des conditions particulières de la visite touristique. Volontairement, on a fait apparaître dans la mission de ce muśe l'objectif de faire aimer Montréal, aux Montréalais évidemment (puisqu'il s'agit du premier grand musce appartenant à la Ville de Montréal et construit avec les taxes des Montréalais) mais aussi, aux touristes. Les concepteurs ont imaginé que tous les touristes passeraient d'abord par la Pointe-à-Callière en arrivant à Montréal parce que ce musée est très bien situé à l'une des entríes du Vieux-Montrél (celle qui est la plus proche de la concentration hôtelière du centre-ville) et aussi, à cause de son propos: l'histoire de la ville, surtout celle des commencements et celle du développement de la principale caractéristique de Montréal: un carrefour d'échanges et de commerce. Une próoccupation constante fut aussi d'intéresser la famille; d'où la salle multimédia, les techniques interactives de communication en sous-sol, les jeux et l'animation à l'exposition permanente dans l'Ancienne Douane, etc.

On a prévu, en fait, que les touristes pourraient explorer à leur rythme l'ensemble des éléments du Musée. Tous les moyens d'interprétation, d' infomation et d'animation sont dis- 
ponibles en français ou en anglais, plusieurs activites sont rapides (exemple: le spectacle d'introduction dans la salle multimédia dure 16 minutes), on a dispose ici et la des bancs pour reprendre des forces ou pour mieux apprécier les artefacts ou les vestiges, etc.

D'autre part, des son ouverture, le Musce de Pointe-ă-Callière a fait entente avec les 5 autres musées et centre d'interprétation du Vieux-Montrial (M.-A. Fortin, Château de Ramesay, Centre d'histoire, Marché Bonsecours et Maison G.-E. Cartier) pour une promotion commune et une concertation thematique. À terme, le pôle du Marché Bonsecours pourrail compléter très bien une visite du VieuxMontrét commenćse à la Pointe-â-Callière (il faudrait maintenant que la vocation de ce Marché Bonsecours se précise évidemment!).

Enfin, même s'il vient à peine d'ouvrir, le nouveau Musée d'archeologie et d'histoire de Montréal travaille avec l'Office des congrès et du tourisme du Grand Montréal, avec les hôteliers, etc. II prendra bientôt sa place comme une grande attraction touristique montrélaise.

\section{Musée d'art contemporain de Montréal}

\section{Madame Louise Faure Relations médias}

Avec sa réouverture au centre-ville de Montréal en mai dernier, le Musce d'art contemporain de Montréal (MAC) s'est donné comme défi d'attirer et de séduire une partie des 35 000 personnes qui défilent quotidiennement dans le couloir qui relie le métro Phace-desArts au Complexe Desjardins, rue SainteCatherine. Au nouveau Musée d'art contemporain, on pense donc grand public, et qui dit grand public, dit bien sûr touristes.

Ces demiers sont comblés cet été avec la crétion d'un forfait, Clabor conjointement avec le Musée des Beaux-Arts de Montréal, le Musce McCord et le Centre canadien d'architecture. Ce forfait, publicisé dans le guide La tournée des grands sites de Montréal permet, à l'achat d'un billet, de visiter ces quatre grands musces montríalais.

Un effort particulier a également été fait cetété pour que le Musće soit présent dans toutes les publications touristiques, comme le guide touristique de Montreal et les publications distribuées dans les hôtels montréalais. La direction des communications et du marketing du $\mathrm{Mu}$ -

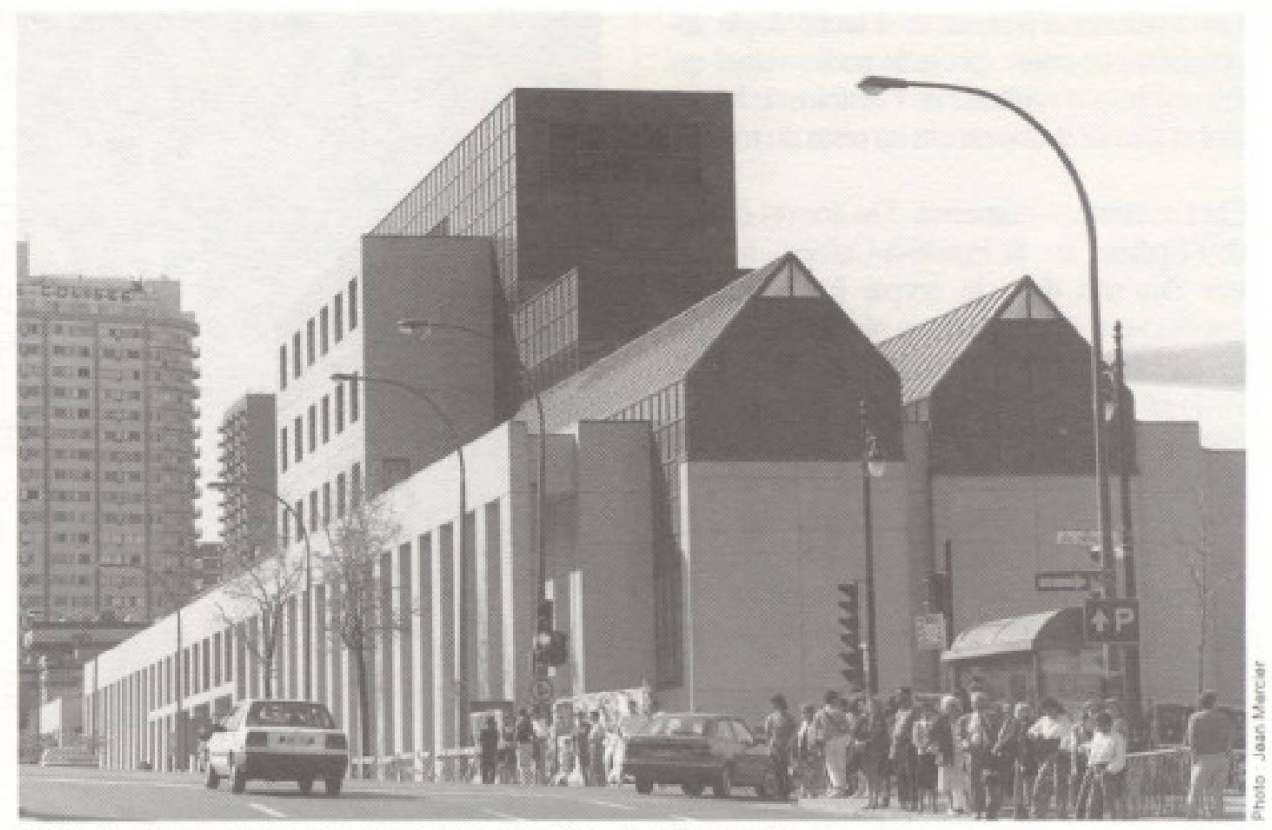

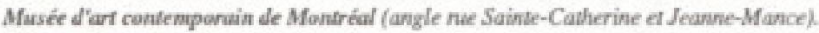

sce, a profité du $350^{\circ}$ anniversaire de Montréal pour augmenter la visibilité du Muséce auprès de la clientèle touristique, afin qu'il soit considéré comme un élément important de leur visite et de leur séjour à Montrél.

Le MAC projette également de concevoir, en collaboration avec les galeries d'art contemporain du centre-ville de Montréal, un dépliant dans lequel serait inclus un plan de la ville, situant l'emplacement du Musée et des galeries d'art. Selon madame Faure, responsable des relations avec les médias au Musce d"art contemporain, ce petit guide pourrait s'averer fort utile aux touristes et autres publics désirant explorer Montral et sa production artistique la plus contemporaine.

Le Musé d'art contemporain n'a certes pas négligé de travailler en étroite collaboration avec l'Office des congres et du tourisme du Grand Montreal (OCTGM). Le Musée veut ainsi assurer sa présence dans le calendrier des événements de l'OCTGM et participer à toutes les activilés que l'Office met sur pied. Par exemple, lors de l'ouverture du Musće, en maí dernier, des journalistes étrangers sont venus à Montrél pour couvrir l'événement. La plupart d'entre eux devant rester plusieurs jours, madame Faure a communiqué avec l'OCTGM afin d'organiser conjointement un tour de ville pour chaque groupe de journalistes. L'Oflice leur a alors offert gratuitement les services de guides touristiques, et en échange, le MAC a distribué aux joumalistes. les pochettes d'informations touristiques sur Montréal et son $350^{\circ}$ anniversaire. Madame Faure qualifie ses relations avec l'OCTGM d'expérience formidable! Le Musce se dit très róceptif et très ouvert afin de mieux connaître le milieu du tourisme et de voir comment il est possible, de part et d'autre, de faire des échanges et d'établir une collaboration plus poussćce. Selon madame Faure, wchaque expérience nouvelle nous amène à une autre idée». Le Musée veut continuer à entretenir des liens avec l'industrie touristique pour favoriser l'effervescence d'idées nouvelles.

Déjà, avant son ouverture, dans la perspective de favoriser des échanges, madame Faure avait rencontré des représentants de l'OCTGM et du ministère du Tourisme afin de mieux cemer et de comprendre les mandats et les responsabilités de chacun. Presque au même moment, en févier, le Musćse d'art contemporain, tout comme le Muste McCord et le Musce de Pointe-à-Callière, recevait 150 guides touristiques afin de leur faire visiter l'institution. Celte rencontre fut, selon madame Faure, très importante puisque ces guides sont des multiplicateurs. D'ailleurs, ils ont acoes gratuitement en tout temps au Musée d'art contemporain.

Un autre intervenant touristique, l'Office du tourisme du Québec a Paris, s'avère une aide très précieuse pour le MAC. II est très actif au niveau médiatique et plusieurs journalistes sont régulièrement invités à se rendre au Québec. C'est ainsi que le MAC accueillera prochainement des journalistes touristiques européens (Partance, Guide du Voyageur, etc.). C'est, selon madame Faure, un moyen très efficace de rejoindre les touristes a l'éranger. 
À un niveau plus régional, le Musće d'art contemporain fait parvenir assez régulièrement aux Associations touristiques régionales de l'information sur le muscée et ses activités, soit des dossiers de presse ou des affiches d'exposition.

Aussi, le Musée d'art contemporain souhaite vivement s'impliquer dans la vie culturelle de Montréal. Ainsi, en raison de son emplacement stratégique sur le site de la Place des Arts, le Musée participeraau prochain Festival International de Jazz de Montréal, qui, à chaque été, est un événement touristique majeur. A cette ocasion, la station de radio CIBL diffusera ses émissions en direct du MAC.

Selon madame Faure, le touriste est le public idéal car c'est une personne en vacances, cunieuse, ayant du temps devant elle. Le public touristique est aussi important que tous les autres publics. Il est même davantage stimulant puisque l'on doit lui expliquer et lui apprendre beaucoup plus. En tant que multiplicateur, il est très important que le touriste soit bien accueilli au musée etait du plaisir à visiter les salles d'expositions. D'ailleurs, les expositions présentées au Musée ont généralement une thématique intemationale, accessible à un public provenant tant de Montréal que de l'extérieur de la ville. Les expositions doivent pouvoir toucher un large public. Toutes les publications disponibles au Musée et les membres du personnel sont bilingues. La dimension touristique est donc très présente à plusieurs niveaux au Musée d"art contemporain de Montréal.

\section{Musée McCord}

\section{Madame Marie-Claire Morin \\ Directrice, \\ Développentent et Communication}

Depuis sa réouverture en mai demier, le Musće MoCord a tenté de se rapprocher davantage de l'industrie touristique. En effet, il existe aujourd' hui une politique et une stratégie de marketing encore plus précise et complète en matière de tourisme.

Plusieurs actions étaient réalisées auparavant, mais aujourd'hui, il y a un effort plus prononcé. aLe tourisme, c'est très important. C"est une industrie, et il faut le traiter comme tel..

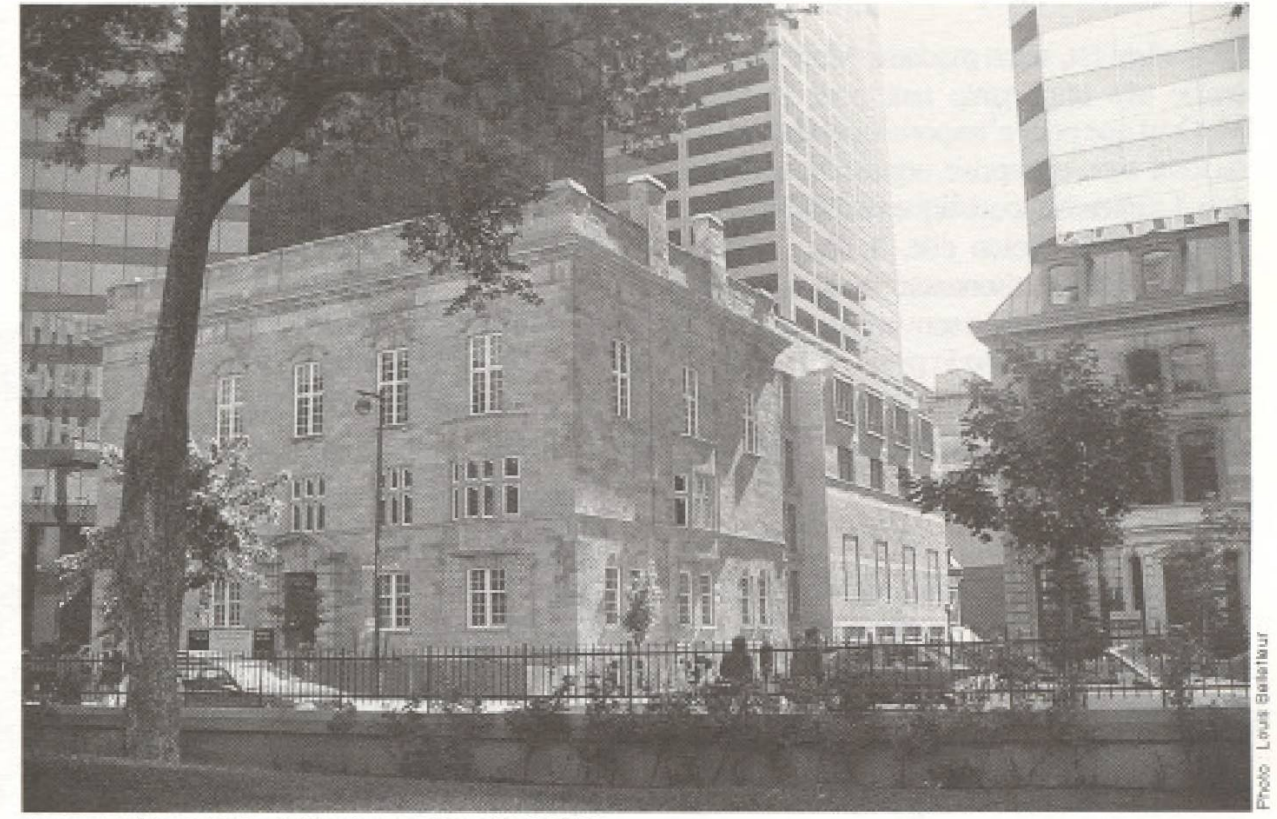

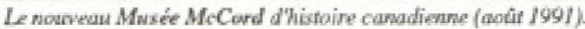

La collaboration du Muscée avec le milieu touristique s'étend au-delà du fait d'être membre de l'OCTGM et d'informer régulièrement le ministène du Tourisme de toutes ses activités. En plus de travailler avec ces deux intervenants majeurs, le Musée McCord entretient d'étroites relations avec les agences de voyages et les voyagistes, qui sont au fait de toutes les activités organisées au Musée, et avec les hơtels de Montréal. Il existe un programme qui vise à mieux faire connaître le Muscée auprès du personnel des établissements hôteliers. Des visites guidées ont donc êté organisées pour ces derniers, et des communiqués de presse ainsi que de la documentation diverse leur sont régulièrement envoyés. Le Musće est aussi très souvent sollicité par les hôteliers désirant obtenir des laissez-passer ou des rabais sur les frais d'admissions, pour une courte durée.

En fait, la direction des communications du Musée MoCord gère une banque de donnćes gui recueille tous les renseignements sur les intervenants touristiques, ces demiers sont tenus au courant de manière périodique et systématique sur tout ce qui se passe au Musée.

Dans le cadre de la réouverture du musée en mai dernier, une invitation avait été lancée à tous les intervenants touristiques de Montreal et de la région, pour mieux leur faire connaître l'institution. Le Musée woulait ainsi leur signifier son intérût ă maintenir et poursuive des échanges avec eux. Selon madame Morin, directrice au développement et aux communications au Musće MoCord, sil y a une très bonne relation et une très bonne collaboration entre le milieu touristique et le Musées.

Le Musée McCord a innové cette année, d'abord en présentant une publicité dans la revue de prestige Presti-Presse, distribuce dans les grands hôtels de Montreal. Ensuite, en publiant une publicité dans le guide La tournée des grands sites de Montréal, dont une section est entièrement consacrée aux quatre grands musées montréalais (Musée MoCord, Musée d Art Contemporain, Musée des Beaux-Arts de Montréal et le Centre Canadien d'Architecture). Produit à un million d'exemplaires, ce guide est distribué au Québec et dans l'est de États-Unis. C'est une promotion très importante pour le Musée McCord qui en est très fier. Une autre nouvelle initiative du Musée a été de faire une publicité conjointe dans des publications spécialisées, entre autres dans des revues de vacances et de voyages, et dans des guides touristiques. $\grave{A}$ propos de ces promotions et publicités produites conjointement avec d'autres institutions muséales, madame Morin explique equ'il est beaucoup plus intéressent pour un visiteur ou un touriste de savoir, lorsqu'il va venir dans la ville qu'il a choisi comme lieu de vacances, qu'il y a trois ou cinq institutions au lieu d'une. Cela rend son choix plus riche et plus important. On pense alors que plus on fait front commun, mieux c'esto.

Le Musée s'assure également d'être continuellement present dans les kiosques de renseignements des hôtels, tout comme dans les plus importants centres d'information touristique du Québec. 
Le touriste est, selon madame Morin, une clientele tress importante tant pour le Musce MoCord que pour n' importe quel autre musce ou toute autre entreprise, organisme ou institution. La clientèle touristique se différencie des autres publics, selon elle, a deux niveaux, D'abord, par sa saisonnalité, et ensuite. par sa curiosité, puisque bien souvent, il ne connaüt ni la ville, ni la région ou le pays qu'il visite. Ses besoins d'informations sur l'histoire canadienne sont alors plus grands et differents de ceux des Québécois ou des Canadiens qui visitent Montréal.

Tout le personnel du Muśe MoCord est bilingue et plusieurs guides sont polyglottes. Le Muśe vise d'ailleurs à développer davantage ce service au fur et à mesure qu'il grandira. Les buts qui sous-tendent ces actions sont a ta fois touristiques el culturels: l'institution veut accucillir le plus de visiteurs possible mais elle desire aussi amélioner, ennichir et soutenir tout ce qui est fait pour loutes les clientèles, incluant la clientèle touristique.

\section{Musée des Beaux-Arts de Montréal}

\section{Madame Suzanne Leblanc \\ Chef du Service des relations publiques}

Le Musće des Beaux-Arts de Montréal est presque un tout nouveau musée depuis l'ouverture, cetteanníe, du Pavillon Jean-Noèl Desmarais au sud de la rue Sherbrooke. Ce nouveau Pavillon - ainsi que le corridor souterraỉn qui le relie à l'ancien - a considérablement agrandi l'espace d'exposition. Et le premier rísullat de oet agrandissement est l'exploitation beaucoup plus considérable de la collection permanente de ce Musée. Madame Leblanc a choisi de rappeler d'abord cette richesse nouvellement révélée du MBAM avant d'aborder les aspects du tourisme. Comme on le verra, les nowveatx produits ont un rapport direct avec les stratégies de services aux clientèles.

Certaines caractéristiques distinguent le Muscé des Beaux-Arts de Montréal des autres musces d'ici et d'ailleurs: il dispose d"abord d'une collection encyclopélique d'oeuvres canadiennes, ce qui lui permet, par exemple, de faire une exposition comme celle qu'on trouve actuellement au Pavillon-nord pour les anniversaires $\left(350^{\circ}\right.$ et $\left.125^{\circ}\right)$, intitulée Nouveaux parcours d'art canadien (artistes montrčalais qui ont marqué notre histoire de l'art:

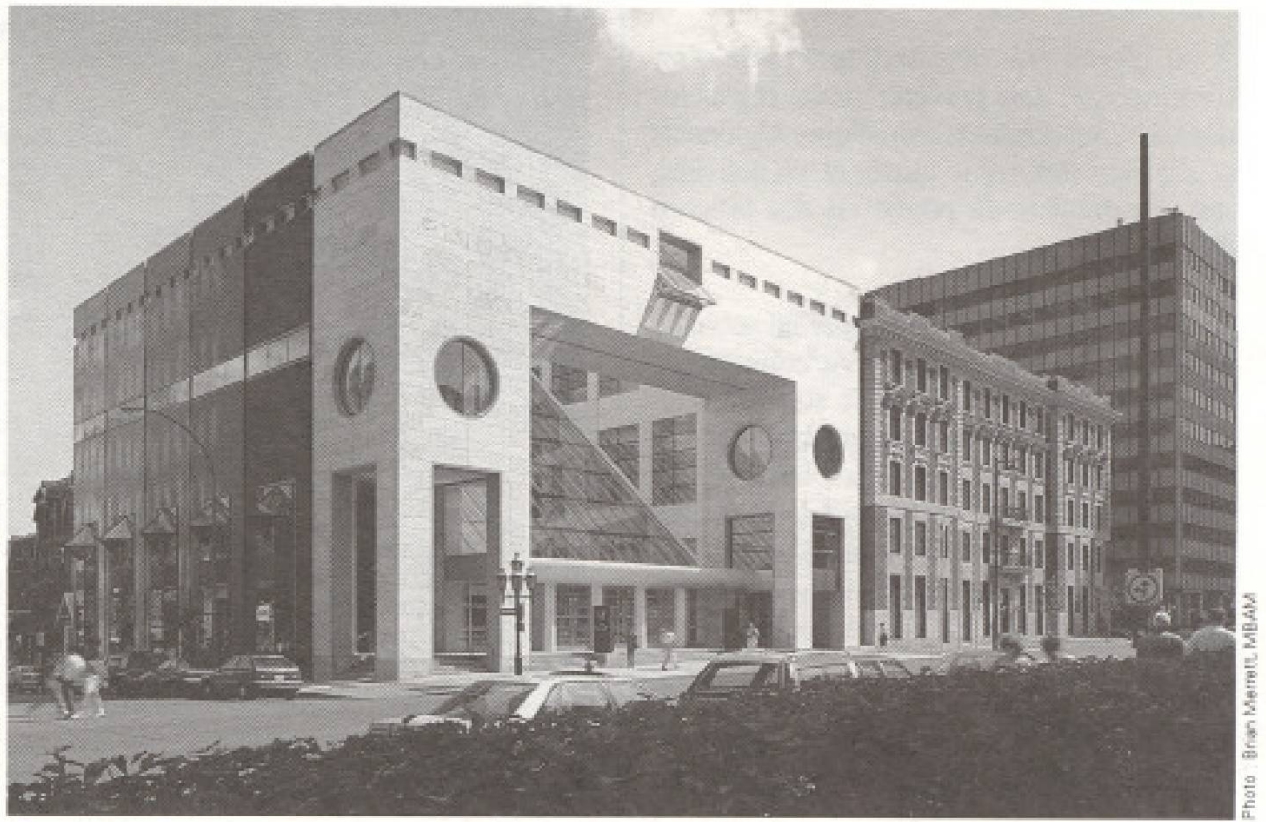

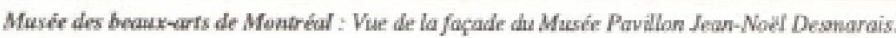

A. Laliberté, J.-W. Morrice et P.-E. Borduas). En second lieu, le MBAM a une grande variété de corpus d'oeuvres de toules les époques, du $X V^{t}$ au $X X^{e}$ siècle, et des conservateurs spécialisés selon ces époques. Enfin, il importe de rappeler que ce Musée a plus de 30 ans d'expérience d'éducation (carrefour, atelier, expositions conrolaires, etc).

Le MBAM peut donc, d'une part, se présenter comme un haut lieu de conservation et d'exposition de la culture artistique d'ici, du Québec et du Canada, par sa collection permanente maintenant plus accessible et aussi, à l'instar d'autres grands musćes, comme un lieu d'exposition d'intérêt intemational qui peut attirer des connaisseurs du monde entier (par exemple, l'exposition Picasso rencontre à Montréal ou enoore, la rícente rétrospective Riopelle, les expositions réussies sur Léonard de Vinci: ingénieur et architecte, sur Marc Chagall, sur les Annuées '20: l'äge des métropoles et celle de cet tế: Le génie du sculpteur dans l'oeuvre de Michel-Ange).

Madame Leblanc profitera de cette exposition en cours consacrée à Michel-Ange, sculpteur, pour présenter les moyens d'interprétation dont dispose le MBAM pour de tels événements. On peut visiter avec guides (en anglais et en français) et avec audio-guides comme dans plusieurs musces; on peut aussi se procurer un court document qui fut largement distribué au début de l'exposition pour s'initier personnellement à l'essentiel du sujet traité. Ce même document suggère un paroours de l'exposition et les temps forts à chaque relais, Enfin, la visite libre reste intéressante parce que le visiteur dispose, devant chaque groupe d'ceuvres, de panneaux didactiques avec étiquettes enrichies pour introduire aux ếments de base de chacune des salles de l'exposition.

Travailler avec l'industrie touristique est un fait acquis pour le MBAM: on offre des visites personnalisées pour les agences de woyages, des prix de groupes, on accueille ces groupes à une entróe spéciale, le Musée fait partie $d^{*}$ une promotion avec trois autres grands musées de Montréal, etc. Dans le plus récent numéro de Collage, la revue du MBAM, son directeur, monsieur Pierre Théberge, signait un texte d'introduction intitule Le musée, attrait touristique numéro un à Montréal (49 139 visiteurs en avril 1992, splus que la fróquentation du Jardin botanique, du funiculaire du Stade olympique et de tous les autres muséesw, p. 2). Le nouveau pavillon venait d'ouvirir, après avoir fait beaucoup parler de lui et présentait une exceptionnelle rétrosnective de Riopelle. L'année 1992 a donc bien commencé pour le nouveau MBAM et l'animation des lieux le 2 juillet, jour de ma visite sous l'habile conduite de madame Leblanc, m'a convaincu que ce musse a atteint une nouvelle vitesse de croisière.

\section{Palais de la Civilisation}

Monsieur Pietro Sicuro Conseiller cadre, recherche et développement

Madame Line Sauwageau Directrice adjointe des expositions 


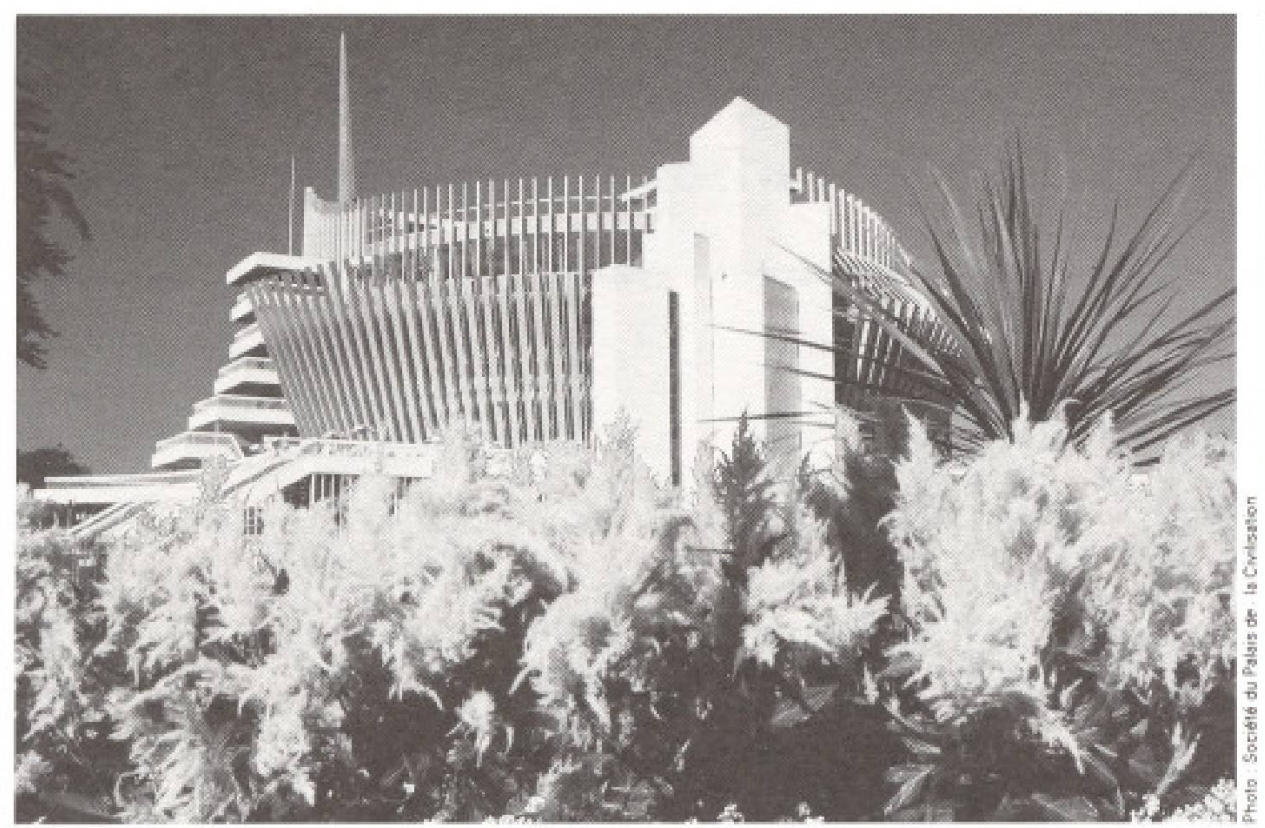

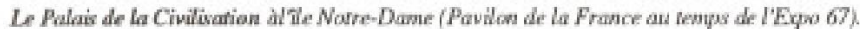

Madane Nathalie Provost Publicite et pronotion

Rome, 1000 ans de civilisation. Tel est le nom de la nouvelle exposition du Palais de la Civilisation qui a róouvert ses portes après une suspension de ses activités de plusicurs mois. Cette institution muscale s' est donnes comme mandat de prisenter des expositions de niveau intemational pour une clientèle très large. $\mathrm{A}$ propos de l'exposition Rome, 1000 ans de civilisation, madame Sauvageau, directrioe adjointe des expositions, explique que apar le contenu et par le mandat il y a ouverture sur le monde, et, par la tenue de cette exposition à Montréal, on a privilégié d'attirer à Montrúal des gens du Québoc et d'ailleurss.

Le type d'exposition qu'on présente au Palais de la Civilisation appelle et attire donc aussi un public touristique. Comme le mentionne $\mathrm{M}$. Sicuro, conseiller cadre à la recherche et au développement, au Palais de la Civilisation, won pense grand public, mais il y a toujours le tourisme en arrière plan... Dans notre philosophie, on inclut le tourisme dans le grand public parce qu'il est partie prenante de ce dernier. La meilleure facon d'intégrer le touriste, $c^{\prime}$ est de lui donner l'illusion qu'il vit au quotidien des autres personnes.....

Le Palais de la Civilisation n'a pas de politiques écrites, à proprement parler, en ce qui oncerne les touristes mais un effort constant est fait pour intéresser et altirer ces demiers. Le marché touristique est l'un de leurs publics cibles et cela se voit. Les exemples sont nombreux. d'art contemporain, permettra aux museses de civilisations d'informer leurs visiteurs sur les expositions présentées dans les autres institutions a travers le monde.

Le Palais de la Civilisation reçoit des subventions du ministère du Tourisme pour toutes les activités dites adirigées en fonction du tourisme \%. A titre d'exemple, une partie de cette subvention leur a permis d'effectuer de la publicité postale sur la côte-est des États-Unis, à Boston et à New York.

Toutes les publications réalisées au Palais de la Civilisation sont bilingues (français et anglais). Généralement, les documents sont aussi publies dans une troisième langue, soit celle de la communauté visée par l'exposition. Par exemple, pour Rome, 1000 ans de civilisation, les dépliants, catalogues (et très prochainement les audio-guides), sont en italien. Cette initiative est très appréciée.

A la boutique, on prend aussi en considération les besoins des touristes. En plus d'y trouver des guides touristiques de Montréal et des cartes postales, les visiteurs peuvent y déoouvrir de nombreux petits objets en relation avec l'exposition pouvant constituer de très beaux souvenirs.

Le Palais s"est également associé récemment au Ritz Carlton et au Musée des Beaux-Arts (exposition Michel-Ange) pour un forfait Venez vivre un été italien à Montréal dont la publicité radiophonique est présentée tant dans la métropole qu'à l'extéricur.

L'institution travaille également en étroite collaboration avec l'Office des congress et du tourisme du Grand Montrial (OCTGM). Elle est présente dans tous les guides de l'Office et elle a pauticiné demièrement à la toumée radiophonique de l'OCTGM qui visitait les principales villes du Québec. On y faisait la promotion des plus importantes attractions de Montréal. A cette occasion, en plus de fournir les principales informations sur l'institution et l'exposition, le Palais de la Civilisation a offert 100 laissez-passer.

Le Palhis de la Civilisation participe aussi très activement à tous les salons touristiques organisés en Amérique du Nord. Il est présent dans plusieurs publications touristiques, entre autres dans le guide Privilegge, dans les brochures et dépliants distribues dans les ctablissements hôteliers, au centre Infolouriste et dans les délégations du Québec à l'étranger. Le Palais participera prochainement à un réseau international appelé Réseau art planète. Ce magazine vidéo, qui existe déjà poư les musćes
Le Palais de la Civilisation đésire maintenir, poursuive et développer sa démarche avec l'industrie touristique. Par exemple, il loue certaines de ses salles pour des réceptions ou des congrès, En septembre prochain, par exemple, le Palais reoevra le congrès de la Fédération internationale des artistes. Aussi, l'institution désire collaborer davantage avee les autres intervenants touristiques situés sur les îles. Il y a, selon madame Provost, responsable de la publicité er de la promotion, aun manque d"unité entre les différents pôles d'attraction sur les îlesw. C'est ainsi que le Palais de la Civilisation $\mathrm{s}^{\dagger}$ associera à des événements tels que la Féerie des lagunes; il offre actuellement des tarifs spéciaux aux personnes qui effectuent la visite des îles par autobus.

Au Palais de la Civilisation, le touniste est important et il est tratité comme tel. Cependant, il ne représente pas un public différent des autres. Il a certes ses particularités mais il ne doit pas être l'objet de privilèges. Comme le mentionne monsieur Sicuro, eon ne pense pas qu'il faut donner un traitement particulier aux. tounistes ou aux autres groupes; on doit tout simplement donner le meilleur traitement pour touss. L'objectif du Palais de la Civilisation: instruire, éduquer et émouvoir tous ses publics. 